\title{
An analytical study of the factors affecting the health promoting lifestyle; comparison of type II diabetic patients with healthy people
}

\author{
Reyhaneh Pourjam $^{\circledR}$, Fatemeh Estebsari ${ }^{*}{ }^{\circledR}$, Zahra Rahimi Khalifeh Kandi ${ }^{3^{\circledR}}$, Kimia Estebsri ${ }^{{ }^{\circledR}}$, \\ Davoud Mostafaei ${ }^{5}$ \\ ${ }^{1}$ Student Research Committee, Department of Midwifery and Reproductive Health, School of Nursing and Midwifery, Shahid Beheshti \\ University of Medical Sciences, Tehran, Iran \\ ${ }^{2}$ Department of Health, School of Nursing \& Midwifery, Shahid Beheshti University of Medical Sciences, Tehran, Iran \\ ${ }^{3}$ Department of Health Education, School of Public Health, Iran University of Medical Sciences, Iran \\ ${ }^{4}$ Maternal Health Expert, Family Health Unit, Azna Health Network, Azna Health Center, Lorestan University of Medical Sciences, \\ Lorestan, Iran \\ ${ }^{5}$ Shahid Beheshti University of Medical Sciences, Tehran, Iran
}

\section{A R T I C L E I N F O}

Article Type:

Original

\section{Article History:}

Received: 10 May 2018

Accepted: 8 July 2018

Published online: 7 August 2019

Keywords:

Lifestyle

Health literacy

Health promotion behavior

Type II diabetes

\begin{abstract}
A B S T R A C T
Introduction: The most important risk factors for type II diabetic patients are poor nutrition, low physical activity, smoking and obesity indicating an inappropriate lifestyle.

Objectives: This study aimed to investigate the factors affecting the health promoting lifestyle in type II diabetic patients compared with healthy people.

Patients and Methods: The study was descriptive-analytic with two case-control groups carried out on 150 type II diabetic patients and 150 healthy people. Data were collected using demographic characteristics questionnaire and health promoting lifestyle profile II (HPLII). Results: There was a significant difference between the lifestyle of type II diabetic patients and healthy people $(P<0.001)$. Additionally, there were significant differences regarding lifestyle dimensions between physical activity, nutrition and stress management in type II diabetic patients compared with healthy people $(P<0.001)$. There was no significant difference between the two groups in the dimensions of spiritual growth $(P=0.074)$, communication with others $(P=0.363)$ and health responsibility $(P=0.582)$.

Conclusion: This study showed the effective role of lifestyle components in the development of type II diabetes. It is recommended to prevent or manage type II diabetes, through emphasizing on maintaining lifestyle and its dimensions.
\end{abstract}

Implication for health policy/practice/research/medical education:

The results of this study showed the effective role of lifestyle components, physical activity and stress management in the development of type II diabetes.

Please cite this paper as: Pourjam R, Estebsari F, Rahimi Khalifeh Kandi Z, Estebsri K, Mostafaei D. An analytical study of the factors affecting the health promoting lifestyle; comparison of type II diabetic patients with healthy people. J Renal Inj Prev. 2019; 8(4): 247-252. DOI: $10.15171 /$ jrip.2019.47.

\section{Introduction}

Chronic non-contagious diseases such as diabetes are the most important causes of mortality worldwide $(1,2)$. The biggest health challenge threating the third world countries is chronic diseases (3). Type II diabetes is recognized as a major public health problem and a health problem of the 21 st century (4). Approximately, $90 \%$ of people with diabetes in the world suffer from type II diabetes $(3,5)$. Global estimates for 2030 predict a 50\% increase in type II diabetes. The number of people with diabetes in 2000 was reported to be $2.8 \%$, and it is expected to reach $4.4 \%$ of the diabetic population by 2030 (1). In 2014, the global prevalence of diabetes in adults over 18 years of age was estimated to be $9 \%$, and in 2012, 1.5 million deaths occurred directly due to diabetes (5).

Diabetes is the fifth cause of mortality in societies and the ninth cause of death in Iranian men $(1,6-8)$. According to the World Health Organization (WHO), in 2000, there were 2,103,000 diabetic patients in Iran, and this number was estimated to increase to 6421000 by 2030 (4). The 
WHO has predicted that diabetes in Iran will involve about $6.8 \%$ of the population by 2025 (3). Additionally, it causes direct costs of $5.2 \%$ to $15 \%$ of the total health budget and indirect costs up to several times (5).

The risk factors for type II diabetes are biological and psychosocial factors. Biologic factors include aging, prevalence of obesity, hypertension and high blood lipids, smoking, alcohol consumption, family history of type II diabetes, insulin resistance, and psychosocial risk factors include environmental risk factors, anxiety and unhealthy food habits (1). The patients with diabetes have multiple physical and psychological disorders such as depression, anxiety, disability, sedentary lifestyle and obesity, which ultimately lead to a decline in their quality of life $(7,9)$. The effects of the disease on social, marital, family, and occupational affairs, spiritual heath and perceived stress affects the quality of life of people with type II diabetes and their families (3). The etiology of type II diabetes is very complicated. Studies have shown that changes in the incidence and prevalence of type II diabetes have occurred in societies with major changes in the type of nourishment experienced by a native diet compared to the Western diet $(1,10)$. The latest research on the cause of type II diabetes has shown that psychosocial factors have a great role in the development of type II diabetes. The most important psychosocial factors are environmental risk factors, low mobility lifestyle, socioeconomic status, gender, ethnicity, low education, poverty, anxiety and unhealthy food habits (1). The most important risk factors for type II diabetes are malnutrition, immobility, smoking and obesity due to an inappropriate lifestyle $(5,7,9,11)$. The effect of lifestyle on the incidence of chronic noncontagious diseases is so important that they are referred to as life-style diseases $(1,12)$. Studies have shown that the incidence of chronic diseases, especially type II diabetes, can be reduced by modified health-promoting behaviors and healthy lifestyle $(9,11,13-15)$. The lifestyle of a person is closely related to their health and the individual performs activities to maintain and improve their health $(9,16$, 17). Nowadays, basic principles of diabetes treatment are diet, physical activity and medicine (3). One of the main target of the WHO is to support effective measures for the care, prevention and control of diabetes and its complications in low- and middle-income countries (5). One of the most effective measures in this area is attention to healthy lifestyle and health-promoting behaviors. Most studies in Iran on type II diabetes etiology have been based on biomedical and clinical approaches. Meanwhile, few studies have been done in Iran on the role of healthpromoting lifestyle and its factors in the incidence of type II diabetes $(1,18)$.

\section{Objectives}

The WHO's motto of 2016 was dedicated to diabetes (19). Considering the importance of diabetes and the knowledge gap in this field, the present study was conducted to investigate the factors affecting the healthpromoting lifestyle of type II diabetic patients compared with healthy peoples. Since the lifestyle of a person is influenced by demographic and socioeconomic variables, these variables are included in the present research. Considering the prevalence of type II diabetes in urban societies, this study was conducted in Tehran as the capital of Iran with the most diverse population.

\section{Patients and Methods}

\section{Study design and patients}

This was a descriptive-analytic study carried out on patients with type II diabetes and their relatives referred to the hospitals affiliated to Shahid Beheshti University of Medical Sciences including Imam Hossein, Shohada, Taleghani and Modarres hospitals that have an active diabetic clinic in 2018. Regarding the effect of age in the development of type II diabetic, patients aged 30 years and older were entered into the study (20). Sampling was conducted systematically based on the last digit of the national code. Inclusion criteria were willing to participate in the study with written informed consent, patients aged 30 years and older, resident in Tehran, a history of at least one year of type II diabetes and having a case in a diabetes clinic, reading and writing skills, lack of mental and psychological health problems and awareness of place and time. Those patients who did not complete the questionnaires were excluded. The number and sample size were obtained using the sample size formula in similar studies (9).

$$
n=2(1.96+1.28)^{2}\left(\frac{1}{0.40}\right)^{2}=132
$$

Taking into account the first error (0.05), type II error $(0.10)$ and power $(0.90)$ and the effect of mean difference in similar studies as 0.40 , the sample size was determined 132 individuals for each group (10). According to 20\% of attrition in each group, the minimum sample size for each group was estimated to be 150 . A total of 300 samples were considered. The questionnaires were completed by participants. Research objectives and the confidentiality of their information were explained for participants. Moreover, they were assured that the data would be used for research purposes and will be published generally.

\section{Data gathering tools}

Data collection tools included two parts; the first part consisted of demographic characteristics including age, gender, marital status (single/married), educational level (elementary/high school/higher), employment status (employed/housekeeper), income adequacy (yes/ no), history of chronic disease (cardiovascular diseases, hypertension, kidney disease or cerebrovascular disease), family history of diabetes (yes/no), health status (how do you assess your health status now? good, moderate and poor), body mass index (BMI) with (normal range; 18.5- 
$24.9 \mathrm{~kg} / \mathrm{m}^{2}$, overweight; $25-29.9 \mathrm{~kg} / \mathrm{m}^{2}$, and obese; > $30 \mathrm{~kg} /$ $\mathrm{m}^{2}$ ), and smoking (yes/no) (21). The question for alcohol consumption was removed because all subjects answered no to the question.

The second part consisted of the Health-Promoting Lifestyle Profile II (HPLP II) questionnaire designed by Pender et al to measure health promoting behaviors and healthy lifestyle (based on Pender's Health-Promotion model) and included 52 items. The questionnaire measures health-promoting behaviors in six dimensions; physical activity (8 items), nutrition (9 items), stress management (8 items), spiritual growth ( 9 items), interpersonal relation (9 items), and health responsibility (9 items). Four-point Likert scale was used ranging from "never" (1 point) to "always" (4 points). The HPLPII score included the total mean score with 6 sub-sets. Higher scores indicated lifestyle-promoting behaviors with a higher frequency and lower scores indicated less frequent behaviors. This tool has been translated into various languages including Persian and its validity and reliability have been verified. In the present study, the reliability coefficient was obtained by Cronbach's alpha 0.86 , which indicates that the questionnaire had a good fit for the present study (22). The reliability of the questionnaire was determined by the repeatability method, in which 25 type II and healthy people were selected by the systematic method. The questionnaires were handed out and completed. Next, the questionnaire was completed by the same individuals after 14 days (23). Finally, the coefficient reliability was calculated. The minimum Cronbach's alpha of 0.75 was considered acceptable. In the present study, the reliability of the questionnaires was confirmed.

\section{Ethical considerations}

Ethical principles regarding human experimentation were in accordance with the Helsinki Declaration 1975, as revised in 1983. The ethical committee of Shahid Beheshti University of Medical Sciences (ethical code; SBMU. RETECH.1396.1244) approved the study. The informed consents were taken from the patients. Besides, this study was extracted from the MSc thesis of Reyhaneh Pourjam (\#13627) at this University.

\section{Statistical analysis}

The collected data were analyzed using SPSS software (version 22) and descriptive statistics (frequency, mean, standard deviation). Inferential statistics (Mann-Whitney $\mathrm{U}$ test, $t$ test and chi-square test) were used for investigating the relationship between the variables. According to the Kolmogorov-Smirnov test, total lifestyle had a normal distribution. Therefore, for comparing the total lifestyle between the two groups, t-test was used. Also, based on the Kolmogorov-Smirnov test, lifestyle dimensions (physical activity, nutrition, stress management, spiritual growth and, interpersonal relation, and health responsibility) did not have a normal distribution. Therefore, the Mann-
Whitney test was used to compare the lifestyle dimensions of both type II diabetics and healthy peoples. In this study, the significance level was considered 0.05 .

\section{Results}

A total of 150 diabetic patients and 150 healthy people were entered into the study. Regarding gender, $58 \%$ of the case (diabetic) group was female. In the healthy group, $63.3 \%$ were female since $36.7 \%$ were male. The mean age in the type II diabetic group was $53.24 \pm 12.28$ years. In the healthy people, it was $46 \pm 11.65$ years. The mean BMI was $29 \pm 3 \mathrm{~kg} / \mathrm{m}^{2}$ in the case group and $24 \pm 2.5 \mathrm{~kg} / \mathrm{m}^{2}$ in the healthy people. Other information is shown in Table 1.

According to the chi-square test, there was a statistically significant difference between gender $(P=0.002)$, education $(P<0.001)$, income adequacy $(P=0.011)$, history of chronic disease $(P<0.001)$, history of diabetes in family $(P=0.003)$, health status $(P<0.001)$, BMI $(P<0.001)$ and smoking $(P<0.001)$ between type II diabetics and healthy people.

The mean and standard deviation of physical activity in the type II diabetic group was $14.75 \pm 3.90$. The results of the Mann-Whitney test to compare the mean scores of physical activities in diabetic and healthy people showed a statistically significant difference between the two groups of the type II diabetics and the healthy people $(P<0.001)$. The mean and standard deviation of the nutritional dimension in the type II diabetic group was 23.35 \pm 3.97 . The results of the Mann-Whitney test to compare the mean nutritional scores showed a significant difference between the two groups of type II diabetics and healthy people $(P<0.001)$. The mean and standard deviation of stress management in the type II diabetic group was $15.47 \pm 3.38$. The results of the Mann-Whitney test showed a significant difference between the two groups, type II diabetes patients and healthy people in terms of stress management $(P<0.001)$. The mean and standard deviation of spiritual growth was $14.75 \pm 3.90$ in the diabetic group. The results of the Mann-Whitney test to compare the mean scores showed, no significant difference in the mean scores of spiritual growths in the two groups of the type II diabetics and the healthy people $(P=0.074)$. The mean and standard deviation of the interpersonal relation in the type II diabetic group was $27.07 \pm 4.74$. The results of the Mann-Whitney test showed, no significant difference between the two groups with regards to interpersonal relation $(P=0.363)$ The mean and standard deviation of health responsibility was $23.96 \pm 4.97$ in the type II diabetic group. The results of the Mann-Whitney test showed, no significant difference between the two groups with regards to health responsibility $(P=0.582)$. Accordingly, the mean and standard deviation of total lifestyle in the diabetic group was $30.58 \pm 17.38$. Meanwhile, the results of the $t$ test showed, a significant difference between the two groups of type II diabetes patients and healthy people regarding total lifestyle variable $(P<0.001)$. 
Table 1. Comparison of frequency demographic variables in type II diabetic patient and healthy people

\begin{tabular}{|c|c|c|c|c|c|c|}
\hline \multirow{2}{*}{ Variables } & & \multicolumn{2}{|c|}{ Diabetic Group } & \multicolumn{2}{|c|}{ Non-Diabetic Group } & \multirow{2}{*}{$P$ value } \\
\hline & & No. & $\%$ & No. & $\%$ & \\
\hline \multirow{2}{*}{ Sex } & Female & 97 & 64 & 95 & 63.3 & \multirow{2}{*}{0.002} \\
\hline & Male & 53 & 36 & 55 & 36.7 & \\
\hline \multirow{2}{*}{ Marital Status } & Single & 22 & 14.8 & 27 & 18 & \multirow{2}{*}{0.450} \\
\hline & Married & 127 & 85.2 & 123 & 82 & \\
\hline \multirow{3}{*}{ Educational level } & Elementary & 38 & 25.3 & 13 & 8.7 & \multirow{3}{*}{$<0.001$} \\
\hline & High school & 50 & 33.3 & 47 & 13.3 & \\
\hline & Higher & 62 & 31.3 & 90 & 60 & \\
\hline \multirow{2}{*}{ Employment status } & Employed & 68 & 45 & 78 & 52 & \multirow{2}{*}{0.444} \\
\hline & Housekeeper & 82 & 55 & 72 & 48 & \\
\hline \multirow{2}{*}{ Income adequacy } & Yes & 62 & 14.3 & 84 & 56 & \multirow{2}{*}{0.011} \\
\hline & No & 88 & 58.7 & 66 & 44 & \\
\hline \multirow{2}{*}{ History of chronic disease } & Yes & 150 & 100 & 45 & 30 & \multirow{2}{*}{$<0.001$} \\
\hline & No & 0 & 0 & 105 & 70 & \\
\hline \multirow{2}{*}{ Family history of diabetes } & Yes & 98 & 65 & 66 & 44 & \multirow{2}{*}{0.003} \\
\hline & No & 42 & 35 & 84 & 56 & \\
\hline \multirow{3}{*}{ Health status } & Good & 30 & 20 & 76 & 50 & \multirow{3}{*}{$<0.001$} \\
\hline & Moderate & 45 & 30 & 47 & 31 & \\
\hline & Poor & 75 & 50 & 27 & 18 & \\
\hline \multirow{3}{*}{ BMI } & Normal & 36 & 24 & 77 & 51 & \multirow{3}{*}{$<0.001$} \\
\hline & Overweight & 71 & 47 & 48 & 32 & \\
\hline & Obese & 42 & 28 & 25 & 16 & \\
\hline \multirow{2}{*}{ Smoking } & Yes & 122 & 81 & 48 & 32 & \multirow{2}{*}{$<0.001$} \\
\hline & No & 28 & 19 & 102 & 68 & \\
\hline
\end{tabular}

Table 2. Comparison of mean square of different dimensions of health-promoting behaviors and total lifestyle in both type II diabetic patients and healthy people

\begin{tabular}{|c|c|c|c|c|}
\hline \multirow{2}{*}{ Variable } & Diabetic group & Non-diabetic group & \multirow{2}{*}{$t$} & \multirow{2}{*}{$P$ value } \\
\hline & Mean \pm SD & Mean \pm SD & & \\
\hline Physical activity & $14.77 \pm 3.90$ & $18.10 \pm 5.19$ & -5.634 & $<0.001$ \\
\hline Nutrition & $23.35 \pm 3.97$ & $26.98 \pm 4.02$ & -7.297 & $<0.001$ \\
\hline Stress management & $15.48 \pm 3.38$ & $21.70 \pm 3.45$ & -12.254 & $<0.001$ \\
\hline Spiritual growth & $25.93 \pm 6.04$ & $27.33 \pm 4.81$ & -1.788 & 0.074 \\
\hline Interpersonal relations & $27.07 \pm 4.74$ & $27.62 \pm 4.20$ & -0.910 & 0.363 \\
\hline Health responsibility & $23.96 \pm 4.97$ & $23.92 \pm 5.80$ & 0.410 & 0.582 \\
\hline Total lifestyle & $130.58 \pm 17.38$ & $145.67 \pm 20.16$ & 6.944 & $<0.001$ \\
\hline
\end{tabular}

\section{Discussion}

In the present study, the mean age of patients with type II diabetes was 53.24 years, the mean age of healthy people was 46 years old. Our results were consistent with the results of other studies. Additionally, people with type II diabetes had a higher mean age than healthy people $(1,20,24)$. Age is one of the effective factors in the occurrence of type II diabetes. Due to the effect of lifestyle and age-related physiological changes, especially in women due to menopause, the incidence and prevalence of type II diabetes increases.

Moreover, based on the results of statistical analysis, gender was an effective factor in the incidence and prevalence of type II diabetes, which was consistent with the results of Estebsari et al and Coumoundouros et al (21, $24)$. In the present study, $87 \%$ of type II diabetic patients were women showing the high prevalence of diabetes in women than men. In the study by Maskarinec et al, the prevalence of type II diabetes in women was high (20).
In the present study, the diabetic group had significantly higher smokers than the healthy people. This is consistent with the findings of Chang's study (25). In the study conducted by Shojaeizadeh et al in 2015, general obesity and abdominal obesity were two important factors for the progression of pre-diabetes among females (26).

In people with lower education, the prevalence of type II diabetes was higher $(21,27,28)$. The higher level of education, the higher the probability that a person will get more information about health and disease from different sources. Hence, health literacy will increase the same proportion and lead to a healthier lifestyle.

The study of lipid and glucose in Tehran found that there is a relationship between smoking and the incidence of diabetes. Azizi's report showed that smoking increases the odds of pre-diabetes up to $70 \%$, which results from the negative effect of smoking on insulin sensitivity and insulin secretion (29). The relationship between a history of smoking and type II diabetes has been confirmed (1). 
The results of this study showed that people with type II diabetes had history of smoking, more disease, lower education, and a history of diabetes in family, which is consistent with the study by Shojaeizadeh et al in 2008 (26).

Regarding the health status, the perceived health status was expressed with the question (how do you assess your health at the moment? Good, moderate, poor). Based on our results, two options, "good" (50\%) and "moderate" (31\%) were found to be higher in healthy people, while the option "poor" was lower in healthy people (18\%) than in type II diabetics (50\%). The studies by Shojaeizadeh et al, Estebsari et al and Tol et al, in 2012 was consistent with the findings of this study $(17,21,27,29,30)$.

Regarding physical activity, the mean score of the healthy people was higher than type II diabetic patients. Some studies were consistent with our results $(1,17,22$, 31 ). Studies have shown that low physical activity is one of the major risk factors for diabetes, since, moderate or high physical activity is associated with a reduction in the risk of type II diabetes $(29,30)$.

Spiritual growth, interpersonal relation, and health responsibility were not significant. In other words, the means for the dimensions of spiritual growth, interpersonal relation, and health responsibility in two groups of type II diabetes and healthy people were similar, which was consistent with the results of the study by Knowler et al (1). Given the fact that healthy people were selected from the healthy relatives of the patients, and that they were related to patients and the hospital staffs, it was likely that these dimensions of lifestyle be of interest to them. Based on the lifestyle health theory, factors such as unhealthy behaviors such as smoking, unhealthy diets and not physical activity lead to an increase in lifestyle risk factors for diseases such as type II diabetes. In addition, major changes in lifestyle such as long-term sitting that leads to the prevalence of obesity and other non-contagious diseases including hypertension and lipid disorders and therefore causes the type II of diabetes. A change in modern life encourages people to take advantage of readymade foods resulting in obesity and type II diabetes. One of the strengths of the present study was the selection of samples from diabetes clinics of large hospitals affiliated to Shahid Beheshti University of Medical Sciences. One of the limitations of this study was using the self-report method to complete lifestyle dimensions questionnaire. Thus, it is recommended to use more direct methods such as measurement of abdominal circumference, hip circumference and biochemical parameters (blood test). Other limitations of this research were using systematic sampling. Because of the conditions of the present study, it was not possible to randomly samples.

\section{Conclusion}

Diabetes is a complex metabolic disease that is considered one of the global challenges. The number of patients with type II diabetes is increasing, which is indicative of a global epidemic. It is recommended that health-based interventions on lifestyle dimensions to prevent and manage type II diabetes emphasizing on factors such as nutrition, physical activity, interpersonal relation, spiritual growth, stress management and health responsibility can be evaluated.

\section{Study limitations}

Low sample size was a limitation of this study. Consequently, randomized controlled trials are necessary to examine the effects of the nutritional, physical activity and stress management in the development of diabetes type II.

\section{Acknowledgments}

This study was related to project No. IR. SBMU. RETECH.1396.1244. The authors would like to thank the "Student Research Committee" and "Research and Technology Chancellor" in Shahid Beheshti University of Medical Sciences for their financial support of this study.

\section{Authors' contribution}

$\mathrm{RP}$ and SFEN designed the study and wrote the manuscript. ZRKK participated in writing the manuscript. KE and DM edited the manuscript before submission. Data analyzing was performed by FE. All authors read and approved the final version and RP and DM submitted the manuscript.

\section{Ethical considerations}

Ethical issues (including plagiarism, double publication) have been completely observed by the authors. This article does not contain any studies with animals performed by any of the authors.

\section{Conflicts of interest}

The research has no conflict of interest.

\section{Funding/support}

The author would like to thank the "Student Research Committee" and "Research and Technology Chancellor" in Shahid Beheshti University of Medical Sciences for their financial support of this study.

\section{References}

1. Knowler WC, Barrett-Connor E, Fowler SE, Hamman RF, Lachin JM, Walker EA, et al. Reduction in the incidence of type 2 diabetes with lifestyle intervention or metformin. $\mathrm{N}$ Engl J Med. 2002;346:393-403. doi: 10.1056/NEJMoa012512.

2. Remington PL, Brownson RC. Fifty years of progress in chronic disease epidemiology and control. Center for Disease Control and Prevention. MMWR. 2011;60:70-7.

3. Ciechanowski PS, Katon WJ, Russo JE. Depression and diabetes: impact of depressive symptoms on adherence, function, and costs. Arch J Med. 2000;160:3278-85.

4. Obirikorang Y, Obirikorang C, Odame Anto E, Acheampong E, Dzah N, Akosah CN, et al. Knowledge and lifestyle- 
associated prevalence of obesity among newly diagnosed type II diabetes mellitus patients attending diabetic clinic at komfo anokye teaching hospital, kumasi, ghana: a hospital-based cross-sectional study. J Diabetes Res. 2016;2016:9759241. doi: 10.1155/2016/9759241.

5. Kang SW, Yoo JS. Health-promoting lifestyle and depression in metabolic syndrome patients in Korea. Int J Nurs Pract. 2012;18:268-74. doi: 10.1111/j.1440-172X.2012.02036.x.

6. Paschalides C, Wearden AJ, Dunkerley R, Bundy C, Davies $\mathrm{R}$, Dickens CM. The associations of anxiety, depression and personal illness representations with glycaemic control and health-related quality of life in patients with type 2 diabetes mellitus. J Psychosom Res. 2004;57:557-64. doi: 10.1016/j. jpsychores.2004.03.006.

7. Fal AM, Jankowska B, Uchmanowicz I, Sen M, Panaszek B, Polanski J. Type 2 diabetes quality of life patients treated with insulin and oral hypoglycemic medication. Acta Diabetol. 2011;48:237-42. doi: 10.1007/s00592-010-0244-y.

8. Costacou T. The Epidemiology of Cardiovascular Disease in Adults with Type 1 Diabetes. Curr Diabetes Rev. 2017;13:520-527. doi: 10.2174/15733998126661609271226 43.

9. Retnakaran R. Novel biomarkers for predicting cardiovascular disease in patients with diabetes. Can J Cardiol. 2018 ;34:624-631. doi: 10.1016/j.cjca.2017.10.017.

10. Payo RM, Mendez XG, Cano CP, Suárez-Álvarez J. Development and Validation of a Questionnaire for Assessing the Characteristics of Diet and Physical Activity in Patients with Type 2 Diabetes. Psicothema. 2018;30:11622. doi: 10.7334/psicothema2017.269.

11. Do Valle Nascimento TM, Resnicow K, Nery M, Brentani A, Kaselitz E, Agrawal P, et al. A Pilot study of a community health agent-led type 2 diabetes self-management program using motivational interviewing-based approaches in a public primary care center in Sao Paulo, Brazil. BMC Health Serv Res. 2017;17:32. doi: 10.1186/s12913-016-1968-3.

12. Bener A, Zirie M, Al-Rikabi A. Genetics, obesity, and environmental risk factors associated with type 2 diabetes. Croat Med J. 2005;46:302-7.

13. American Diabetes Association. Lifestyle management: standards of medical care in diabetesd-2018. Diabetes Care. 2018;41:38-50. doi: 10.2337/dc18-S004.

14. Darkhor S, Estebsari F, Hosseini M, Yazdani Charati J, Vasli P. Effect of health promotion intervention on Nurses' healthy lifestyle and health-promoting behaviors: RCT study. J Adv Pharm Edu Res. 2018;8:108-114.

15. Steyn NP, Mann J, Bennett PH, Temple N, Zimmet P, Tuomilehto J, et al. Diet, nutrition and the prevention of type 2 diabetes. Public Health Nutr. 2004;7:147-65.

16. Babazadeh T, Kiani A, Moradi F, Shariat F, Banaye Jeddi M. The Effect of self-care behaviors education on quality of life in patients with type 2 diabetes: a randomized clinical trial. J Fasa Uni Med Sci. 2017;6:538-47.

17. Estebsari F. The Effect of Educational Intervention Program on Physical Activity. J Paramed Fac Tehran Univ Med Sci. 2008;2:56-63.

18. Rahimi Foroushani A, Estebsari F, Mostafaei D, Eftekhar Ardebili H, Shojaeizadeh D, Dastoorpour M, et al. The effect of health promoting intervention on healthy lifestyle and social support in elders: a clinical trial study. Iran Red Crescent Med J. 2014;16:e18399. doi: 10.5812/ircmj.18399.

19. Noncommunicable Disease Prevention and Health Promotion. http://www.who.int/hpr/about.ncds.shtml. Accessed October 4, 2003.

20. Maskarinec G, Jacobs S, Park SY, Haiman CA, Setiawan VW, Wilkens LR, et al. Type II diabetes, obesity, and breast cancer risk: the Multiethnic Cohort. Cancer Epidemiology and Prevention Biomarkers. 2017;26:854-61. doi: 10.1158/10559965.EPI-16-0789.

21. Estebsari F, Shojaeizadeh D, Mostafaei D, Farahbakhsh M. Planning and Evaluation of an Educational Program Based on PRECEDE Model to Improve Physical Activity in Female Students. J Faculty Nurs Midwife. 2010;16:48-54.

22. Estebsari F, Taghdisi MH, Rahimi Foroushani A, Eftekhar Ardebili H, Shojaeizadeh D. An educational program based on the successful aging approach on health-promoting behaviors in the elderly: a clinical trial study. Iran Red Crescent Med J. 2014;16:e16314. doi: 10.5812/ircmj.16314.

23. Johansson L, Silén M. Research methods in nursing students' Bachelor's theses in Sweden: A descriptive study. Nurse Educ Today.2018;66:187-93. doi: 10.1016/j.nedt.2018.04.006.

24. Coumoundouros C, Ould Brahim L, Lambert SD, McCusker J. The direct and indirect financial costs of informal cancer care: A scoping review. Health Soc Care Community. 2019;00:1-15. doi: 10.1111/hsc.12808.

25. Chang SA. Smoking and type 2 diabetes mellitus. Diabet Metabol J. 2012;36:399-403.

26. Shojaeizadeh D, Estebsari F, Aazam K, Batebi A, Mostafaei D. Comparison of Factors Affecting Lifestyle in Patients with Type II Diabetes and Risk Factors in Shahid Rajaee Hospital in Tonekabon, 2005. J Shahid Sadoughi Uni Med Sci Health. 2008;16:71-9.

27. Piatti P, Setola E, Galluccio E, Costa S, Fontana B, Stuccillo M, et al. Smoking is associated with impaired glucose regulation and a decrease in insulin sensitivity and the disposition index in first-degree relatives of type 2 diabetes subjects independently of the presence of metabolic syndrome. Acta Diabetol. 2014;51:793-9. doi: 10.1007/s00592-014-0599-6.

28. Tol A, Pourreza A, Tavasoli E, Rahimi Foroshani A. Determination of knowledge and health literacy among women with type 2 diabetes in teaching hospitals of TUMS. J Hosp. 2012;11:45-52.

29. Derakhshan A, Sardarinia M, Khalili A, Momenan AA, Azizi F, Hadaegh F. Sex specific incidence rates of type 2 diabetes and its risk factors over 9 years of follow-up: Tehran Lipid and Glucose Study. PLoS One. 2014;9:e102563. doi: 10.1371/journal.pone.0102563.

30. Kiajamali M, Hosseini M, Estebsari F, Nasiri M, Ashktorab T, Abdi AH, et al. Correlation between social support, selfefficacy and health-promoting behavior in hemodialysis patients hospitalized in Karaj in 2015. Electron Physician. 2017;9:4820-4827. doi: 10.19082/4820.

31. Abdi J, Eftekhar H, Estebsari F, Sadeghi R. Theory-based interventions in physical activity: a systematic review of literature in Iran. Glob J Health Sci. 2014;7:215-29. doi: 10.5539/gjhs.v7n3p215.

Copyright $\odot 2019$ The Author(s); Published by Nickan Research Institute. This is an open-access article distributed under the terms of the Creative Commons Attribution License (http://creativecommons.org/licenses/by/4.0), which permits unrestricted use, distribution, and reproduction in any medium, provided the original work is properly cited. 